\section{STRUCTURE WATCH}

\section{A stimulating structure}

Follicle-stimulating hormone (FSH) - a glycoprotein hormone that comprises a common $\alpha$-subunit and a hormone-specific $\beta$-subunit — is key to mammalian reproduction. It binds to the G-protein-coupled receptor FSHR on target cells and induces testicular and ovarian functions. To further understand this interaction, Fan and Hendrickson now describe the 2.9- $\AA$ resolution crystal structure of partially deglycosylated FSH bound to the hormone-binding domain of FSHR $\left(\mathrm{FSHR}_{\mathrm{HB}}\right)$ in Nature. $\mathrm{FSHR}_{\mathrm{HB}}$ is mainly composed of leucine-rich repeats, and these were found to wind up to form a curved tube. The central region of FSH binds to the concave face of this tube in a 'hand-clasp' manner. The interaction interface is large $\left(2,600 \AA^{2}\right)$, with a high charge density, and their data indicate that this mode of binding is of relevance to all mammalian glycoprotein-hormone-receptor complexes. The authors analysed the interface contacts for determinants of specificity, and identified three key interaction sites on FSHR that vary among the different receptors and that contact residues that vary among the different hormones. These interaction sites involve both the $\alpha$ - and $\beta$-subunits of the hormones. They also found that, on binding $\mathrm{FSHR}_{\mathrm{HB}}$, FSH undergoes a conformational change, which rigidifies the protruding loops that are thought to be involved in receptor activation. In addition, $\mathrm{FSH}-\mathrm{FSHR}_{\mathrm{HB}}$ formed dimers, both in the crystals and at high concentrations in solution, so it will be interesting to determine whether dimerization is required for signal transduction.

REFERENCE Fan, Q. R. \& Hendrickson, W. A. Structure of human follicle-stimulating hormone in complex with its receptor. Nature 433, 269-277 (2005)

\section{Robust rings}

For chromosomes to be accurately segregated to daughter cells, spindle microtubules must become attached to kinetochores multiprotein complexes that assemble on centromeric DNA. One kinetochore component in Saccharomyces cerevisiae is the tensubunit DASH complex, and mutations in this complex disrupt kinetochore-microtubule attachments and destabilize the spindle. New insights into this complex are now provided by Harrison and colleagues in Nature Structural \& Molecular Biology.

They co-expressed the entire yeast complex in Escherichia coli, and purified a $\sim 210-\mathrm{kDa}$ decamer that contained one copy of each subunit. The hydrodynamic properties of recombinant DASH indicated that it is representative of the in vivo complex. Electron microscopy (EM) studies showed that DASH is a globular complex, which oligomerizes to form closed rings and paired helices only in the presence of microtubules. These rings/helices encircle microtubules, although, in the EM images, there seemed to be a gap between the rings/helices and the microtubule. Harrison and co-workers therefore propose that extended polypeptides might project from DASH to contact the microtubule (such extensions might not be visible in these images). In addition, as helices only form at high DASH concentrations, they propose that it is the rings that are biologically relevant, and that rings are used to ensure that the kinetochores stay attached to dynamic microtubules.

REFerence Miranda, J. L. et al. The yeast DASH complex forms closed rings on microtubules. Nature Struct. Mol. Biol. 12, 138-143 (2005)

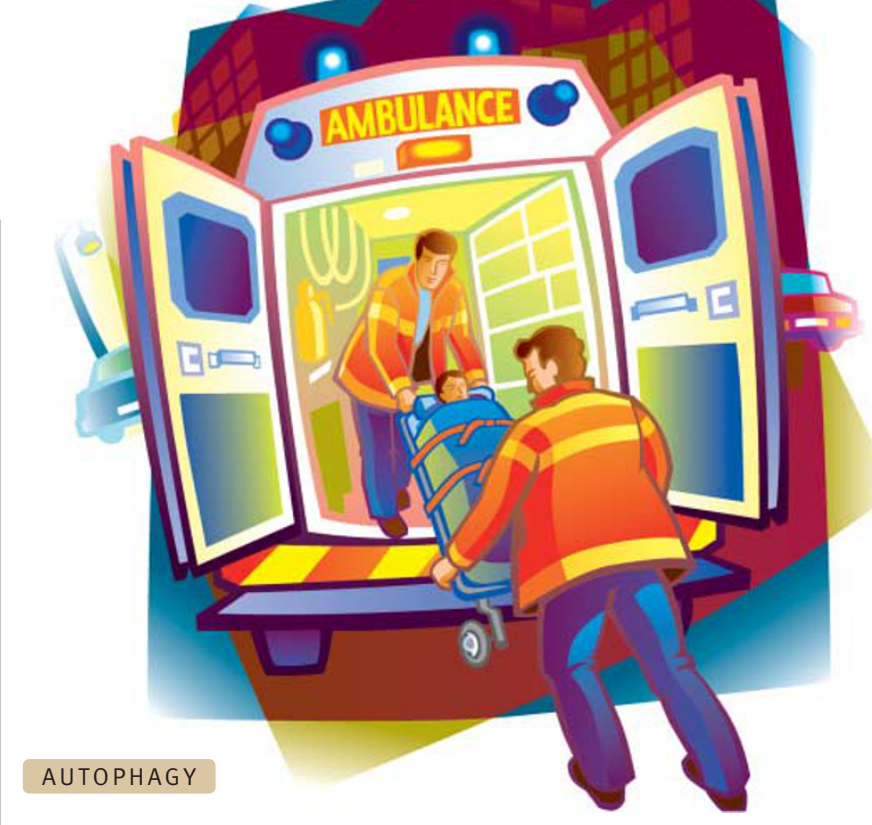

\section{Survival factor}

Animal cells need growth factors and nutrients for their survival. When depleted of growth factors, cells undergo apoptosis, whereas nutrient starvation leads to a process known as macroautophagy, during which basal ATP production is maintained by catabolizing intracellular substrates, to allow short-term cell survival. Reporting their findings in Cell, Craig Thompson and colleagues now show that, even in the presence of excess nutrients, apoptosis-deficient cells that are deprived of growth factors undergo macroautophagy.

Using cells from interleukin (IL)-3-dependent $\mathrm{Bax}^{-/} \mathrm{Bak}^{-/-}$mice, which are apoptosis deficient, Thompson and co-workers noticed that cells shrank in size in response to IL-3 withdrawal and showed a prolonged survival until, after $\sim 12$ weeks, they started to die. Cells also showed metabolic changes, including reduced glycolysis and a drop in ATP levels, as well as reduced cell-surface expression of the nutrient transporter GLUT1 and the formation of autophagosomes, which are characteristic of macroautophagy.

To analyse whether macroautophagy was responsible for the survival of growth-factor-deprived cells, Thompson and colleagues inhibited autophagy by RNA interference - after which cells indeed started to die. When cells that had been deprived of IL-3 for several weeks were treated with macroautophagy inhibitors, cell death could be reversed by supplying cells with an alternative metabolic substrate to maintain oxidative phosphorylation and, therefore, ATP production. This shows that the continued degradation of metabolic substrates is important to maintain cell viability, even at late time points after growth-factor withdrawal. Importantly, macroautophagy was induced by growth-factor withdrawal, and not by a lack of nutrients, as the IL-3-depleted cells were kept in rich culture medium.

Next, the Thompson group showed that when IL-3-deprived cells were resupplied with IL-3, the rate of glycolysis increased within hours, which reflects their ability to take up and metabolize glucose again. The recovery time for cells to start growing and proliferating took longer, and depended on the length of IL-3 deprivation. Virtually all cells that were deprived of IL-3 for 2 or 6 weeks recovered, so growth-factor-mediated signal transduction is ultimately required for cell survival.

The authors concluded that, in addition to regulating apoptosis, growth factors seem to promote cell survival by enabling cells to take up sufficient nutrients for limited self-maintenance. This dual role makes them essential survival factors.

Arianne Heinrichs

(1) References and links

ORIGINAL RESEARCH PAPER Lum, J. J. et al. Growth factor regulation of autophagy and cell survival in the absence of apoptosis. Cell 120, 237-248 (2005) 